\title{
The implications of bent jets in galaxy groups
}

\author{
Danielle M. Nielsen and Eric M. Wilcots \\ Dept. of Astronomy, University of Wisconsin - Madison \\ 475 N. Charter Street, Madison, WI, 53706, USA \\ email: nielsen@astro.wisc.edu
}

\begin{abstract}
Bent-double sources, sometimes referred to as wide- or narrow-angle tails, are most likely the result of ram pressure from either the motion of the source through a dense medium or the "cluster weather." These unusual sources have long been associated with high density, high velocity dispersion, and turbulent environments of massive clusters, however a surprising number have been found in lower mass systems. We focus our attention on a sample of such sources in galaxy groups where the velocity dispersion is significantly lower. We have acquired multi-frequency radio continuum observations using the GMRT of our bent-double sample and new optical spectroscopy to measure the velocity dispersion of groups hosting these bent-double sources. Our goal is to derive an estimate of the density of the intergalactic medium in these groups. Here, we present GMRT data for one source in our sample.
\end{abstract}

Keywords. intergalactic medium, radio continuum: galaxies

\section{Introduction}

We have taken a novel approach to measuring the density of the intergalactic medium (IGM) and baryonic content of galaxy groups through its effect on the jets of radio galaxies (Freeland et al. 2008; Freeland \& Wilcots 2011). As a double lobed radio source travels through the intergalactic gas, ram pressure causes its jets to be swept back. Although it had been thought that these sources require a dense IGM only found in rich clusters, a surprising number have been found in lower mass systems. Freeland \& Wilcots (2011) derive IGM densities in the range of $10^{-4}-10^{-3} \mathrm{~cm}^{-3}$ for a small sample of groups. They also place an upper limit of $2 \times 10^{6} \mathrm{~K}$ on the temperature of the IGM for two groups, a factor of 5-10 less than typical temperatures of hot gas observed in X-ray detected groups. The work discussed here expands on this previous work of Freeland \& Wilcots (2011) by expanding the sample size of bent-double sources and more thoroughly measuring the jet properties.

\section{Methods \& Data}

We use Euler's equation and a measure of the radius of curvature of the jet to estimate the ram pressure acting on the jet (Venkatesan et al. 1994). Euler's equation describes the balance of internal and external pressure gradients for a non-relativistic, hydrodynamic jet:

$$
\frac{\rho_{\mathrm{IGM}} v_{g}^{2}}{h}=\frac{\rho_{j} v_{j}^{2}}{R}
$$

Here, $\rho_{\text {IGM }} v_{g}^{2}$ is the external ram pressure, $\rho_{j} v_{j}^{2}$ is the jet ram pressure, $h$ is the radius of the jet, and $R$ is the radius of curvature. High resolution radio continuum observations are used to measure $\rho_{j} v_{j}^{2}, R$, and $h$. We equate the velocity of the source as the difference 


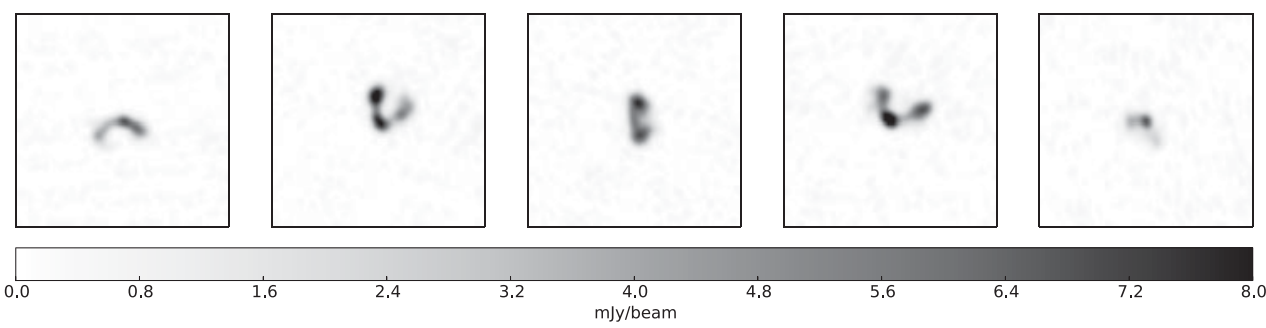

Figure 1. FIRST survey cutouts of five bent-double sources in our sample which we have observed with the GMRT and VLBA.
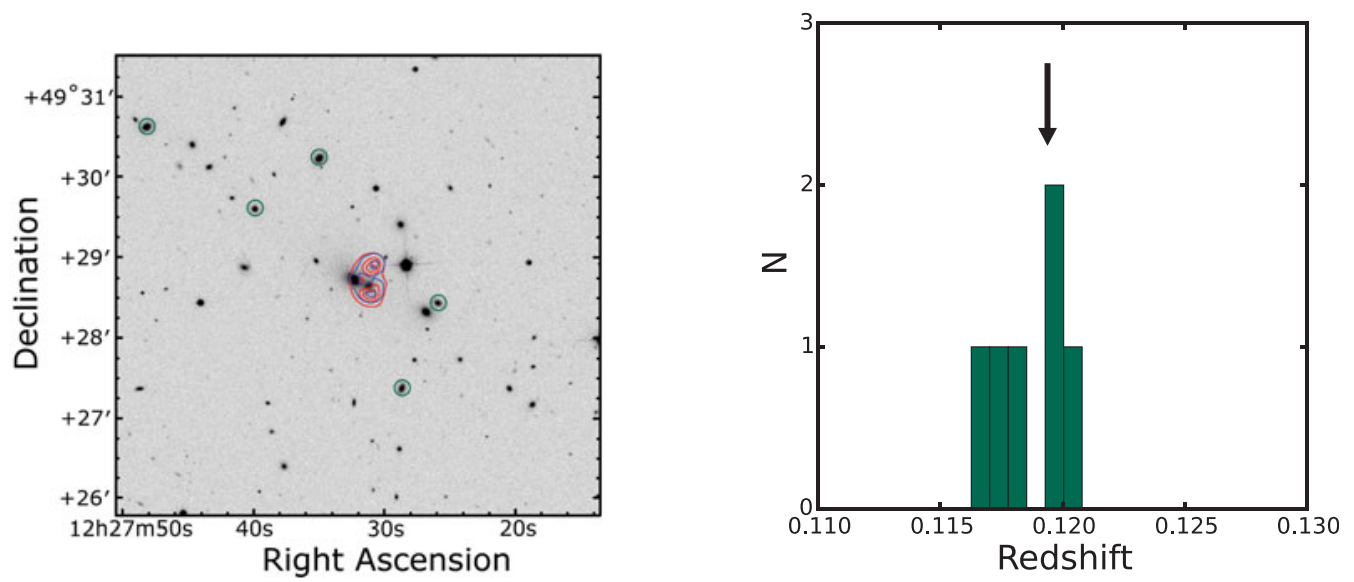

Figure 2. Left: Radio contours of a bent double source from our sample overlaid on a SDSS optical image. Red contours were created from GMRT observations at $610 \mathrm{MHz}$. Blue contours are $1.4 \mathrm{GHz}$ FIRST survey data. Neighboring galaxies with SDSS spectroscopic redshifts are circled in green. Right: Shown is a velocity distribution created from SDSS spectroscopic redshifts for the bent-double source and neighboring galaxies. The redshift of the bent-double source is indicated by the black arrow.

between its velocity and the mean velocity of the surrounding galaxies which requires accurately measuring the velocity distribution of the environment.

We compiled a sample of bent-double sources in poor environments identified from the FIRST survey by Wing \& Blanton (2011) to which we will apply this analysis. FIRST survey cutouts of five sources in our sample are shown in Fig. 1. We have obtained radio continuum images with the GMRT and are carrying out spectroscopic observations with SALT, WIYN, and Gemini-North. Radio contours of one source in our sample and a preliminary velocity distribution are shown in Fig. 2.

The largest source of uncertainty in our analysis is the assumptions of jet properties for our sources. We have recently obtained VLBA observations at 6 and $18 \mathrm{~cm}$ of our sample galaxies to directly measure the radius and spectral index of the jet. Additionally, we will determine where the bending of the jet begins with respect to the host galaxy.

\section{References}

Freeland, E., Cardoso, R. F., \& Wilcots, E. 2008, ApJ, 685, 858

Freeland, E. \& Wilcots, E. 2011, ApJ, 738, 145

Venkatesan, T. C. A. et al., 1994, ApJ, 436, 67

Wing, J. D. \& Blanton, E. L. 2011, $A J, 141,88$ 